\title{
Computer Assisted Validation of using newly Introduced Thermoplastic Material in Fabrication of a Telescopic Denture
}

\author{
May U. Hamzah \\ Biomaterials Department \\ Faculty of Oral and Dental \\ Medicine \\ Cairo University \\ Giza, Egypt
}

\author{
Ahmed M. Sayed \\ Biomedical Engineering \\ Department \\ Faculty of Engineering, Helwan \\ Helwan University \\ Cairo, Egypt
}

\author{
Tamer M. Nassef \\ Computer and Software \\ Department \\ Faculty of Engineering \\ MUST University \\ Giza, Egypt
}

\begin{abstract}
This study aims to analyzing the validity of using a newly introduced thermoplastic over denture material, by estimating the stress distribution, resultant strains and failure locations within the complete telescopic denture, using a three dimensional finite element model. Mainly two materials were tested and compared with each other; an acrylic resin with a Co$\mathrm{Cr}$ alloy and an acrylic resin with a flexible acetal resin, with the later being the new material under testing. The finite element model was implemented based on real data from CT scans. For model validation purposes, ten over dentures were constructed and subjected to experimental fracture tests. Results of modeling method were realistic, and showed a relatively lower over denture stresses using the acetal frameworks compared with those using the Co-Cr framework. In summary, the outcomes of the proposed finite element model were found to be in good agreement with the mechanical testing measurements, which would verify using this model for the evaluation of new dental materials.
\end{abstract}

\section{Keywords}

3D Finite element analysis, modeling, telescopic over dentures, acetal resin

\section{INTRODUCTION}

Rehabilitation of a partially edentulous patient can be established using a wide range of prosthetic treatment options depending upon the clinical needs and patients' demands. Polymethyl methacrylate (PMMA) was introduced in 1937 as a prosthetic treatment material. Since then, it became the most popular polymer material used in dentistry. The low cost, good aesthetics, ease of fabrication, manipulation and polishing has made PMMA a preferred denture base material $[1,2]$.

With the increasing dental awareness and elderly population, it is reasonable to expect that more and more patients will require various dental prosthetic treatment options. Classic removable dentures are still an important treatment option for completely or partially edentulous patients. However, telescopic dentures with the aid of its retentive capacity provide a more suitable prosthetic treatment. Conventionally, telescopic dentures are made of acrylic resin and metal framework to ensure good aesthetics and mechanical properties [3].
An optimum restoration depends on the clinician's skills in the selection of material type and restoration design for each clinical situation. The fabrication of prosthesis for completely or partially edentulous arches encounters special challenges when soft tissue and bony undercuts interfere with various paths of placement. Tilted teeth and unbalanced occlusion are considered additional complications for treatment planning $[4,5]$.

Recent advancements in material science motivated by the increased patient's esthetic demands and anxiety due to metallic allergies and metallic bad esthetics led to the development of metal-free prosthesis. Flexible denture base materials have been developed in 1950 and underwent several improvements to meet such demands [6, 7]. However, limited information is available on the stress distribution encountered when these flexible materials are used in combination with conventional acrylic resin complete over dentures [8]. Therefore, computer biomechanical models are suggested to study such stress distributions, and the method of finite element modeling has proved to be a powerful tool of modern engineering science to study such complex problems [9].

This study investigates the validity of using a thermoplastic material in the fabrication of telescopic dentures, using the developed stress distributions and related information obtained from the described computer model. The stress distributions were estimated under different loading schemes through finite element modeling.

\section{MATERIALS AND METHODS}

A typical Finite element modeling (FEM) analysis primarily consists of three stages; preprocessing, processing, and postprocessing. The preprocessing stage consists of geometry building, incorporating material properties, creating the mesh, applying boundary conditions, and finally applying loads. The processing stage consists of solving a set of equations simultaneously to obtain the nodal variables; displacements. The governing equations were structural bio-mechanical equations, which are imbedded in the ANSYS software. The postprocessing stage involves calculating the resultant stresses, and also the graphical representation of the solution $[9,10]$. 


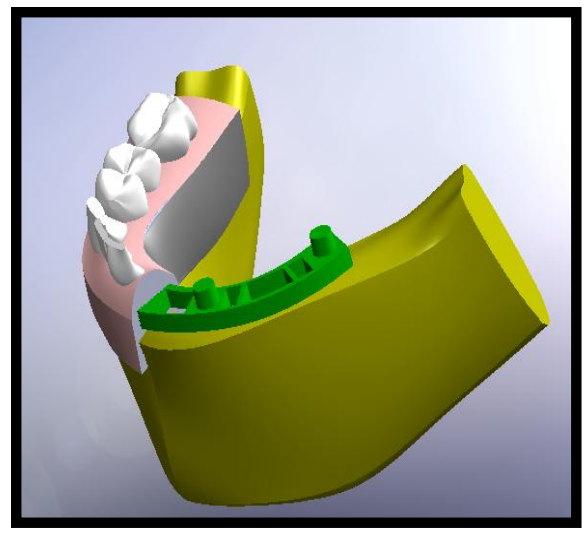

a)

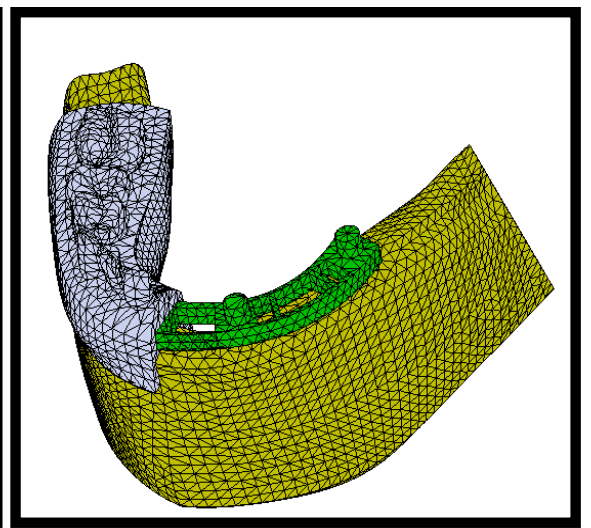

b)

Fig .1: a) Geometric model's Components. b) Meshed model.

The whole finite element analysis in this study was executed using a personal computer (I5 processor, 4GB RAM). Three software packages were employed in this study for model construction. The first software was Image Materialise Mimics (Materialise, Leuven, Belgium) for selecting the region of interest from dental CT scans, where the patients were wearing their over dentures. The INUS Rapidform XOR3 (INUS technology, Inc, USA) was used for the 3D model construction. The third software was Solid works 2012 (Solid Works Corp., Concord, MA, USA) for performing the FEM analysis. The following subsections describe the finite element analysis in detail.

\subsection{Creation of the geometrical model}

A number of steps were performed inside the mimics software starting by separating the hard (bone and denture) from the soft tissue by selecting appropriate density values from sample CT scans based on value thresholding; the detailed steps were described in a previous study [11].

Different zones in the region of interest were identified through the selection of a range of grayscale values (654 to 2070). This was followed by building the 3D model's geometry to form an STL (stereolithographic) model upon which the CAD (computer aided design) model was drawn.

Then, the CAD model was introduced into an intermediate software (rapidform) to draw specific curves representing the mandibular body, denture base and teeth. Following that, the curves were connected to draw the mandibular body, then four circles were drawn protruded at the assumed positions of the dental implants at the sites of the canine and the first molar bilaterally.

Using the same approach, the denture base and teeth curves were then connected. The lingual flange of the over denture was drawn apart from the rest of the over denture. This was performed to allow the use of different materials' properties.

A plane was drawn parallel to the crest to represent the framework by dividing the distance from the cervix of the teeth to the bone crest. Four circles were used to represent the implant position. The circles were built out from the denture side and cut through from the bone side to accept the implant abutments emerging from the mandibular model. Then, the components were assembled together using the common points between them, as shown in figure (1.a).

At this stage, the modeling process continues by the meshing the model's geometry; dividing the problem space into finite elements. An approximate solution for the equations governing the behavior of elements can be developed for the whole object, taking into consideration the linkage between the elemental solutions to ensure continuity at the boundaries. The main element type used in this study was structural tetrahedral element. Thus, The final meshed model is shown in figure (1.b).

Two different models were constructed using the described loading method. Model I: telescopic mandibular over denture formed from conventional heat-cured acrylic resin and cobaltchromium alloy framework supported by the mandibular bone. Model II: telescopic mandibular over denture formed from conventional heat-cured acrylic resin and acetal (thermoplastic flexible) framework supported by the mandibular bone.

\subsection{Introducing material properties in the model}

All used materials in the model were considered to be homogenous, isotropic and linearly elastic. The moduli of elasticity and Poisson's ratios of the studied materials were tabulated in tables I and II [12].

Table 1. Materials properties of the various supporting structures used in this study [12]

\begin{tabular}{l|l|l}
\hline Materials & $\begin{array}{l}\text { Elastic Modulus } \\
(\mathbf{M P a})\end{array}$ & Poisson's ratio \\
\hline Cortical bone & 13.700 & 0.3 \\
\hline Trabecular bone & 1370 & 0.3 \\
\hline Oral Mucosa & 680 & 0.45 \\
\hline Titanium implants & 103.400 & 0.35 \\
\hline Copper & 110.000 & 0.34 \\
\hline
\end{tabular}

Table 2. Materials properties of the telescopic over dentures components in the different models [12]

\begin{tabular}{l|l|l}
\hline Materials & $\begin{array}{l}\text { Elastic } \\
\text { Modulus } \\
\text { (MPa) }\end{array}$ & $\begin{array}{l}\text { Poisson's } \\
\text { ratio }\end{array}$ \\
\hline Acrylic resin tooth & 3000 & 0.35 \\
\hline
\end{tabular}




\begin{tabular}{l|l|l}
\hline $\begin{array}{l}\text { Conventional heat- } \\
\text { cured acrylic resin }\end{array}$ & 3000 & 0.35 \\
\hline Acetal resin & 2800 & 0.35 \\
\hline $\begin{array}{l}\text { Cobalt- chromium } \\
\text { alloy }\end{array}$ & 218.000 & 0.33 \\
\hline
\end{tabular}

As a final step in the preprocessing phase, bonded contact elements were used to prevent penetration or separation between elements representing different anatomical parts of the mandible.

\subsection{Stress analysis}

The loading scheme was realized using occlusal pressure to make the stresses very near the points of application. The value of the applied pressure was $150 \mathrm{~N} / \mathrm{mm} 2$; average human occlusal pressure. More amount of deformation in these points was obtained with minimal distribution to the neighboring parts. Following the previous steps, the FEM model was run; processing phase, for each model and the resultant nodal displacement were used to calculate strains and hence the resultant stresses.

\subsection{Mechanical testing}

Ten over dentures were constructed. The over dentures were divided into two main groups (for each group, $n=5$ ) according to the combination of materials used in their construction. Five of each over denture at a crosshead speed of $0.5 \mathrm{~mm} / \mathrm{min}$ until fracture. This attachment was used to ensure proper stress distribution on the over denture.

\section{RESULTS \\ 3.1 FEM analysis}

In Model I (telescopic over denture made from acrylic resin/Co$\mathrm{Cr}$ supported by bone), the maximum stresses were found to be concentrated mainly at the lingual flange bilaterally including the anterior region with an amount of $120 \mathrm{MPa}$ (shown in figure 2 with a red color). The stress concentration was seen with a lower degree in the cervical area of the first, second premolars and first molar (yellow to green color with less red). These stresses were more observed on the lingual side than the buccal side of these teeth especially on the first premolar. Minimum stresses $0 \mathrm{MPa}$ were found in the buccal flange of the incisor area (blue color) except in the area of the midline which was color coded with green denoting moderate stresses in this area.

In model II (telescopic over denture made from conventional heat-cured acrylic resin/Acetal supported by bone), the maximum stresses were found to be concentrated mainly at the cervical area around the first premolars bilaterally with an amount of $110 \mathrm{MPa}$ (color coded with an orange to red color in figure 3). Additionally, stress concentration was found to be lower in the cervical area of the second premolar and first molar (color coded mainly with a yellow to green color and less red). Minimum stresses $(0 \mathrm{MPa}$ ) were found in the incisor area (color coded with a blue color). The lingual flange of the anterior

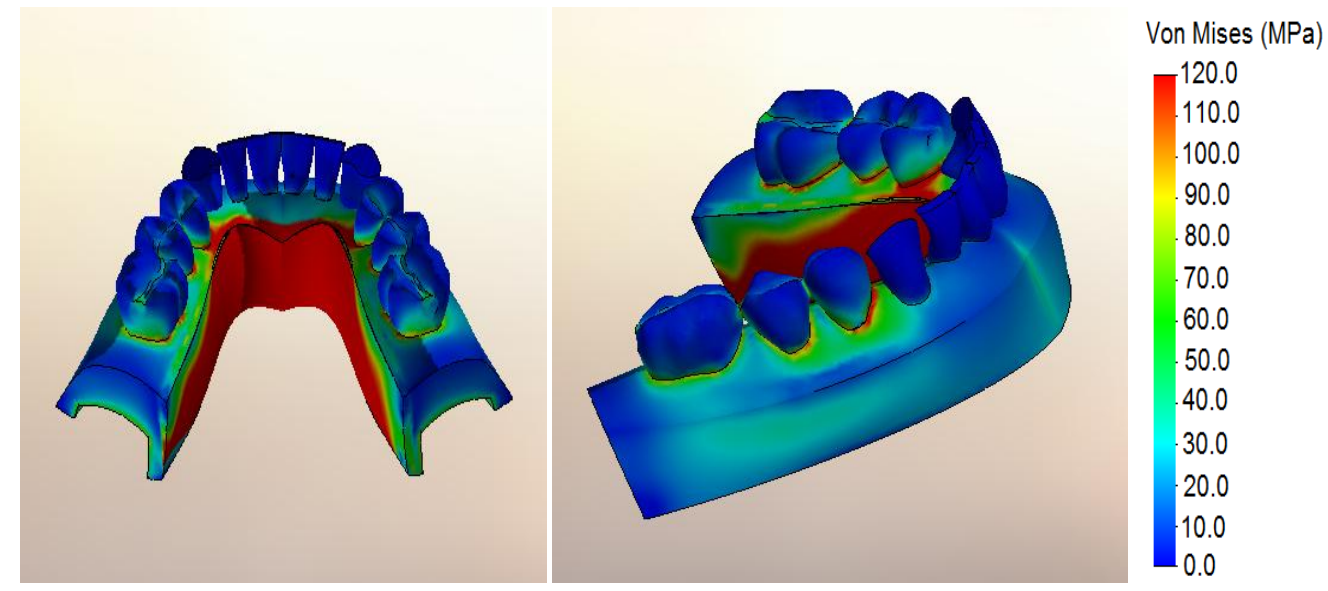

Fig. 2: Maximum Von mises in AR/ Co-Cr alloy framework supported by bone; model I

these over dentures were fabricated from conventional heatcured acrylic resin with a flexible acetal resin (Polyoxymethylene) framework (test group). The other five over dentures were fabricated from conventional heat-cured acrylic resin with $\mathrm{Co}-\mathrm{Cr}$ alloy framework (control group).

Each telescopic complete over dentures was subjected to fracture resistance test using a universal testing machine (LR5K, LLoyd instruments, LTD, England). The load was applied through a horse-shoe shaped attachment on the occlusal plane of region of the over denture showed a moderate stresses ranging approximately from $40-90 \mathrm{MPa}$ which were coded by yellow to green color.

It can be observed from the demonstrated FEM results, that the maximum stress values were very close in the studied models, with slightly higher maximum stresses of $120 \mathrm{MPa}$ observed in the acrylic resin/Co-Cr material, rather than the $110 \mathrm{MPa}$ observed in the acrylic resin/Acetal material. This observation was confirmed by the statistical analysis of the mechanical tests. 


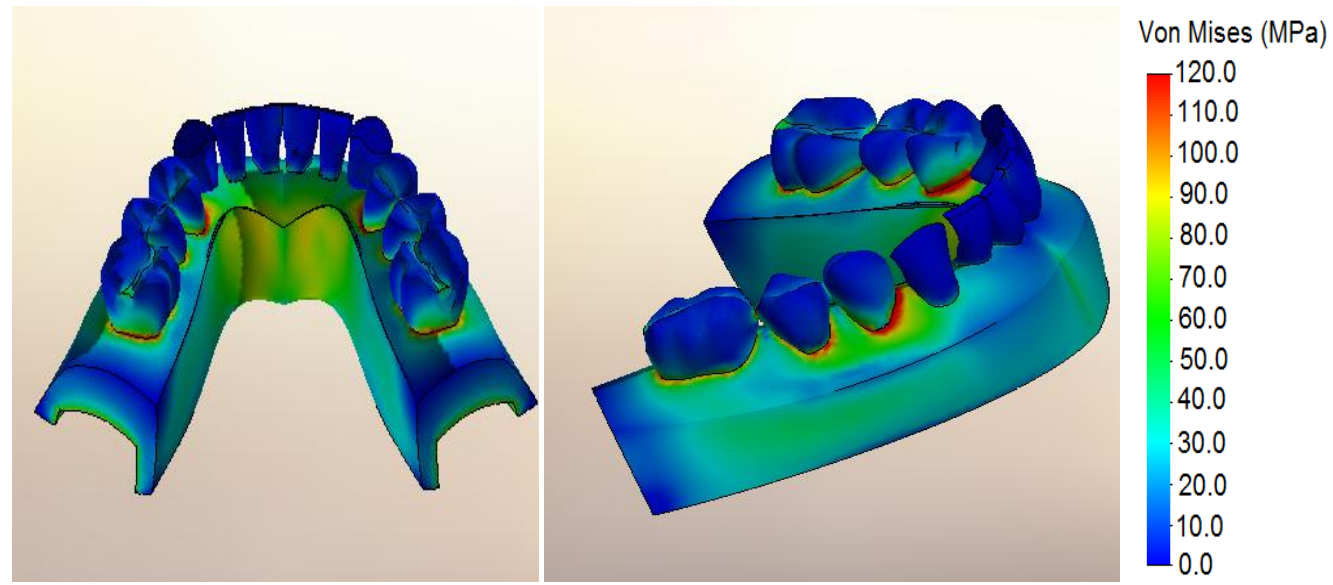

Fig. 3: Maximum Von mises stresses in AR /Acetal framework supported by bone; model II.

\subsection{Fracture test results}

The results of the fracture load of the investigated telescopic complete over dentures made from conventional acrylic resin with $\mathrm{Co}-\mathrm{Cr}$ alloy frameworks and those made of conventional acrylic resin with flexible acetal resin frameworks are given in Table III. The five acrylic resin/Co-Cr over dentures fractured adjacent to the abutments, while the five acrylic resin/Acetal over dentures fractured adjacent to the abutments and framework. The mean loads at failure were $3450.1 \pm 722.3 \mathrm{~N}$ and $2958.2 \pm 703.7 \mathrm{~N}$ for acrylic resin/Co-Cr alloy framework and acrylic resin/Acetal resin framework telescopic complete over dentures, respectively. One-way Analysis of Variance (ANOVA) was used to test the differences between the investigated materials. Tukey's post-hoc test was used for pairwise comparison between the groups when ANOVA test was significant. The significance level was set at $\mathrm{P} \leq 0.05$. It was found that no statistically significant differences were existing between the two groups, as the calculated P-value was equal to 0.337. Therefore, the estimated locations and values of the maximum stress values from the FEM analysis were coincide and in agreement with the failure locations and relative values found in laboratory tests.

\section{DISCUSSION}

In this paper, it was noticed that the maximum Von mises stresses within the telescopic over denture made from heat-cured acrylic resin and $\mathrm{Co}-\mathrm{Cr}$ alloy framework when supported by bone and/or copper models were $120 \mathrm{MPa}$. These stresses were concentrated in the whole lingual flange of the over denture and to lower extent on the cervical areas of the posterior teeth. On the other hand, the maximum Von mises stresses developed within the telescopic over denture made from heat-cured acrylic resin and flexible resin framework when supported by bone and/or copper models were $110 \mathrm{MPa}$. These stresses were concentrated at the cervical area of the posterior mainly the first premolars bilaterally. The relatively lower Von mises stress value generated within the over denture with the acetal frameworks (110 MPa) could be related to the dissipation ability of stresses offered by the higher flexibility of the acetal resin. However, this higher flexibility (lower elastic modulus of 2800 $\mathrm{MPa}$ ) allowed higher stress concentration than the rigid $\mathrm{Co}-\mathrm{Cr}$ alloy framework (218.000 MPa). Additionally, the general lingual concentration of stresses on both cases could be attributed to the lingual inclination of the posterior teeth [13].
Table 3. Fracture load of both groups

\begin{tabular}{|c|c|c|c|c|}
\hline \multicolumn{2}{|c|}{$\begin{array}{l}\text { Acrylic resin with } \\
\text { Co-Cr }\end{array} \begin{array}{l}\text { alloy } \\
\text { framework } \\
\text { (AR+ alloy) }\end{array}$} & \multicolumn{2}{|c|}{$\begin{array}{l}\text { Acrylic resin with } \\
\text { flexible acetal resin } \\
\text { framework } \\
\text { (AR+ Thermoplastic) }\end{array}$} & \multirow[t]{2}{*}{$\begin{array}{l}\text { P- } \\
\text { value }\end{array}$} \\
\hline $\operatorname{Mean}(\mathrm{N})$ & SD & $\operatorname{Mean}(\mathrm{N})$ & SD & \\
\hline 3450.1 & 722.3 & 2958.2 & 703.7 & 0.337 \\
\hline
\end{tabular}

Furthermore, the maximum stresses related to the first molar region decreased gradually in the distal part, to reach its minimum value at retro-molar pad and anteriorly toward the incisors. This may be due to the rotation of over denture around implant, which acts as a fulcrum $[8,14]$

The results of mechanical testing revealed that there was no significant difference of the fracture load among the two types of telescopic over denture; conventional heat-cured acrylic resin with acetal resin framework, and conventional heat-cured acrylic resin with $\mathrm{Co}-\mathrm{Cr}$ alloy framework. This could be caused by the lower strength properties and low crack initiation and propagation as compared to $\mathrm{Co}-\mathrm{Cr}$ alloy, in addition to the brittle nature of the conventional heat-cured acrylic resin (tensile strength of about $48.3 \mathrm{MPa})[15,16]$ as compared to the acetal resin (tensile strength of about $70 \mathrm{MPa}$ ), which would degrade its fracture toughness.

Furthermore, the method of fabrication could play a role in the final strength properties of the resins. The injection molding technique used with acetal resins has been proven to improve the propertied of the processed resins. This method exclude mixture errors, decrease porosities, maximize the degree of conversion as compared to the compaction molding technique used with the conventional heat-cured acrylic resin.

The fracture lines of all tested over dentures occurred adjacent to the abutment. This could be attributed to the fact that during loading the over dentures displaced downward where the abutments would function like a fulcrum. Additionally, the denture thickness was reduced in the area around or adjacent to the abutments [17]. Thus weakening of the denture base could occur at these sites.

\section{CONCLUSIONS}

Based on the demonstrated results, it can be concluded that the use of finite element analysis is availed and agree with the mechanical testing of telescopic over dentures. Also, the use of bone as support during finite element analysis provided a more 
realistic clinical simulation. In addition, it was found that Cobalt-Chromium alloy framework produced better stress distribution within over dentures than acetal frameworks, although the acetal framework could allow for a wider range of displacements of the over denture than Cobalt-Chromium alloy framework. The model provided a valuable tool to estimate the material behavior under stress. Future work includes computational testing of other new materials to find the optimal design for a telescopic denture.

\section{REFERENCES}

[1] S. Bayer, et al., "Retention force measurement of telescopic crowns," Clin Oral Investig, vol. 14, pp. 607-11, Oct 2010.

[2] G. Tandon R., Agarwal S.K, "Denture base materials: From past future," Indian Journal of Dental Science, vol. 2, pp. $33-39,2010$

[3] K. C. Baher M., Lang R.,Hahnel S.,Dirschl L., Dent C.: , "Clinical performance of cements as luting agent for telescopic double crown retained removable partial and complete overdentures," International Journal of Prosthodontics Dentistry, vol. 22, pp. 479-87, 2009.

[4] C. A. Dodge, "Prevention of complete denture problems by use of over dentures," Journal of Dental Restoration, vol. 30, pp. 403-411, 1979.

[5] Ahmed M Sayed, et al., "Quantitative Assessment of Gingival Inflammation Using High-Resolution Ultrasound Ex-Vivo," Journal of Mechanics in Medicine and Biology, vol. 18, 2018/4/16 2018 .

[6] C. B. Lavinia Ardelean, Angela Podariu and Laura Rusu, Manufacture of Different Types of Thermoplastic, 2012.

[7] MM Elramady, et al., "Measuring primary stability for the inclined implants retaining mandibular overdenture using resonance frequency," presented at the Cairo International Biomedical Engineering Conference (CIBEC), Cairo 2014.

[8] M. Daas, et al., "A complete finite element model of a mandibular implant-retained overdenture with two implants: comparison between rigid and resilient attachment configurations," Med Eng Phys, vol. 30, pp. 218-25, Mar 2008.

[9] D. V. Hutton, Fundamentals of finite element analysis, International ed. Boston: McGraw-Hill, 2004.

[10] A. A. H. Sayed, et al., "Finite element models for computer simulation of intrastromal photorefractive keratectomy," Journal of Mechanics in Medicine and Biology vol. 11, pp. 1255-1270, 2011

[11] T. M. Nassef, "New Segmentation Approach to Extract Human Mandible Bones Based on Actual Computed Tomography Data," American Journal of Biomedical Engineering, vol. 2, pp. 197-201, 2012.

[12] Y. E. Kayabas O., Erzincanl F, "Static, dynamic and fatigue behaviors of dental implant using finite element method," Advances in Engineering software, vol. 37, pp. 649-658, 2006.

[13] R. S. Fajardo, et al., "The effect of E-glass fibers and acrylic resin thickness on fracture load in a simulated implant-supported overdenture prosthesis," J Prosthet Dent, vol. 106, pp. 373-7, Dec 2011.

[14] A. Erdemir, et al., "Considerations for reporting finite element analysis studies in biomechanics," J Biomech, vol. 45, pp. 625-33, Feb 232012.

[15] H. A.M, "The Fracture Pattern of Different Mandibular Over-Denture Designs," Al-Rafidain Dent, vol. 1, 2008.

[16] M. Rosentritt, et al., "Fracture performance of computeraided manufactured zirconia and alloy crowns," Quintessence Int, vol. 40, pp. 655-62, Sep 2009.

[17] M. Behr, et al., "Clinical performance of cast clasp-retained removable partial dentures: a retrospective study," Int J Prosthodont, vol. 25, pp. 138-44, Mar-Apr 2012. 